\title{
THE CONTEXTUAL INTERFERENCE EFFECT ON THE PERFORMANCE OF FUNDAMENTAL MOTOR SKILLS IN ADULTS
}

original paper

( ) University School of Physical Education in Wrocław

DOI: https://doi.org/10.5114/hm.2018.73608

\author{
JUDITH JIMENEZ-DIAZ ${ }^{1}$, MARIA MORERA-CASTRO ${ }^{2}$, WALTER SALAZAR ${ }^{1}$ \\ ${ }^{1}$ University of Costa Rica, San Pedro, Costa Rica \\ ${ }^{2}$ National University of Costa Rica, Heredia, Costa Rica
}

\section{ABSTRACT}

Purpose. The aim of the study was to analyse the effect of contextual interference on acquisition and retention for jump and throw in adults.

Methods. The participants $(n=50)$ were randomly assigned to 3 groups: blocked practice (BP), random practice (RP), and control group (CG). During each practice session, the BP group performed 20 trials of one skill, followed by 20 trials of the second skill, while the RP group performed 20 trials for each skill in a random order. The CG participated in physical activities that did not include any of these two skills. The intervention consisted of 7 sessions. Skill performance was assessed with the Test of Fundamental Motor Skills for Adults - for pretest, acquisition, and retention. The test has content validity established by logical validity, as well as documented intra-class reliability (calculated via test-retest) and inter-rater reliability.

Results. A two-way ANOVA [group (3) $\times$ measurement (3)] with repeated measures in the last factor revealed a significant interaction in throw $(F=5.81 ; p=0.001)$ and jump $(F=10.92 ; p=0.001)$. Post-hoc analyses indicated that the BP and RP groups improved from pretest to acquisition. The CG was statistically significantly different from the experimental groups in the acquisition and retention phase. The RP and BP groups were not statistically different in any phase, both of the skills being assessed.

Conclusion. No contextual interference effect on fundamental motor skills in adults was found. Nonetheless, the results suggest that RP and BP improved performance for both skills.

Key words: motor learning, distance jump, overarm throw, blocked practice, random practice

\section{Introduction}

The manner in which the physical practice of a skill is scheduled can affect the performance in acquisition, retention, and/or transfer [1, 2]. Practice can be structured promoting a high contextual interference with random practice - where the trials of the task to be learned are organized in an alternated sequence (e.g. A, B, A, B, A, A...). Or, practice can be prepared to promote low contextual interference - with blocked practice - where trials are arranged in a consecutive sequence (e.g. A, A, A, A..., B, B, B, B...) [1, 3]. When organizing practice under different levels of contextual interference, immediate and long-term performance can be enhanced or disrupted. This learning phenomenon is best known as the contextual interference effect (CIE) [4-6].

The CIE predicts that practicing under low contextual interference enhances acquisition, but leads to poorer performance on retention and transfer tests as compared with practicing under high contextual interference, and vice versa $[1,6,7]$.

The typical CIE prediction has been found in sequence motor learning in undergraduate students $[8$, 9]. Wegman [10] also observed the effect on a fundamental motor skill (FMS) in middle school girls. Contrarily, the effect was not established in the field hockey skills of undergraduate students [11, 12], in throwing tasks in elementary school students [13], or in sport-related tasks among older adults [14]. Evidence

Correspondence address: Judith Jimenez-Diaz, Universidad de Costa Rica, 11-501-2060, Montes de Oca, San José, Costa Rica, e-mail: judith.jimenez_d@ucr.ac.cr

Received: July 10, 2017

Accepted for publication: November 22, 2017

Citation: Jimenez-Diaz J, Morera-Castro M, Salazar W. The contextual interference effect on the performance of fundamental motor skills in adults. Hum Mov. 2018;19(1):20-25; doi: https://doi.org/10.5114/hm.2018.73608. 
has shown consistency in the prediction of the CIE, especially in laboratory-based studies $[15,16]$. Nonetheless, applied research has brought mixed results $[10,11,13,15,17]$.

Furthermore, meta-analytic results prove that blocked practice is bound with better performance in acquisition compared with random practice $[16,18]$. In retention tests, Mazzardo [18] found that random practice showed better performance than blocked practice, but Jiménez-Díaz et al. [16] observed no significant difference between groups. Both meta-analytic studies revealed that the age of the participants, the number of trials, the external validity of the study, and the type of skill are factors that moderated the CIE $[5,16,18]$.

In addition, evidence has shown a high prevalence, in children and adolescents, of low competency in FMS [19]. The little evidence available with reference to adults also revealed low motor competency in these skills [20]. As motor competency is associated with physical fitness in adults [21], it is necessary to enhance FMS performance in this population. A meta-analysis showed that performance of FMS can be improved in children with motor interventions [22], but evidence on motor interventions to enhance FMS in adults is limited.

Therefore, this study has two purposes: to examine the CIE on FMS in adults and to determine the effect of random and blocked practice on FMS performance.

\section{Material and methods}

\section{Participants}

The total of 61 students were initially recruited for this experimental study; 4 participants dropped out during practice sessions for personal reasons and $7 \mathrm{did}$ not attend the acquisition or retention tests. Therefore, the final sample consisted of 50 undergraduate students (38 male and 12 female), who volunteered to participate in this field study (age: $20.32 \pm 2.07$ years; height: $1.68 \pm 0.08 \mathrm{~m}$; weight: $63.93 \pm 0.08 \mathrm{~kg}$; body mass index [(BMI]: $\left.22.62 \pm 3.40 \mathrm{~kg} / \mathrm{m}^{2}\right)$. The study received institutional and ethical approval, and the participants signed the informed consent.

All participants were healthy students enrolled in a physical activity program at a university in Costa Rica. None of the students attended Physical Education, Human Movement Sciences, or similar majors.

\section{Instruments and measurements}

Motor skill performance for distance jump and overarm throw was assessed with the Test of Fundamental Motor Skills for Adults (TFMSA) [23]. The TFMSA is a process-oriented test that consists of 2 subscales: locomotor (run, gallop, slide, distance jump, and hop) and object control (catch, kick, strike, overarm throw, and bounce), 10 FMS in total. For this study, we used the distance jump and overarm throw scales. Each skill is described by 6 observable components (Table 1 )

Table 1. Observable components to assess FMS proficiency

\begin{tabular}{ll}
\hline FMS & Observable components \\
\hline Distance & Preparatory movements include flexion of both knees, the trunk is slightly inclined forward, and arms \\
jump & extended behind the body above the hips without crossing the middle line of the body. \\
& In takeoff, arms extend forward and upward and are kept fully extended above the head during the flight. \\
In takeoff, the body is fully extended. \\
Takeoff and landing are performed with both feet simultaneously. \\
Knees are bent for landing to soften it. \\
Arms swing downward and forward during landing. \\
Overarm \\
throw & Preparatory movements include one foot forward and the other backward. The body weight is on the back foot \\
& of the same side as the throwing arm. \\
& During preparation, trunk rotates substantially toward the side of release. \\
In throwing, a defined rotation of the hips and shoulders is given. \\
During preparation, the throwing arm is moved backwards (elbow flexed) when the launch takes off, the arm \\
is moved forward with the elbow flexed, and extends completely in the front to release the object. \\
During preparation, the opposite arm is pointing toward the direction where the object must be released. \\
During the launch, it is carried toward the body. \\
After releasing the object, the back foot is brought to the front, changing the body support.
\end{tabular}

Translated from the original instrument (Spanish to English) [23].

FMS - fundamental motor skill 
that together constitute proficiency. Each participant was videotaped when performing 2 trials for each skill, and they received 1 point if the component of the movement was present or 0 points if not. Performance was assessed from the videotape footage. The raw score for each skill ranged from 0 to 12 points. The total of 9 or more points indicates a proficient performance. For the distance jump, the documented scale intraclass reliability (calculated via test-retest) is 0.82 and the inter-rater reliability equals 0.73 ; for the overarm throw, the intra-class reliability is 0.92 and the interrater reliability 0.73 [23]. The TFMSA was applied to assess throw and jump performance at pretest, acquisition, and retention.

In addition, height and weight were measured for the participants' descriptive information. Height was measured with a portable measuring board (Seca 213). A bioelectrical impedance scale (OMRON HBF-510LA) was used to measure body weight.

\section{Experimental groups and procedures}

The study consisted of 10 sessions: 1 for the pretest, 7 for the intervention, 1 for the acquisition test, and 1 for the retention test.

On the first session, the participants signed the consent form and filled out the personal information. Then, they were measured for height $(\mathrm{cm})$ and weight $(\mathrm{kg})$; BMI was calculated with the standard formula and expressed in $\mathrm{kg} / \mathrm{m}^{2}$. After collecting height and weight, the students were assessed for pretest on motor skills performance (jump and throw), as indicated in the test protocol. They were videotaped when performing 2 trials of each motor skill and the performance was assessed from the videotape footage. After the pretest, the participants were randomly assigned to 3 groups: blocked practice, random practice, or control group.

During the acquisition phase, the participants attended 7 sessions ( 2 sessions in each of the first 3 weeks; 1 session in the $4^{\text {th }}$ week), of 30 minutes each. For practice, they performed 20 trials for each skill per session, for the total of 140 trials for each skill. The practice trials were not assessed. The blocked practice group (low contextual interference) performed 20 consecutive trials of one skill, followed by 20 consecutive trials of the second skill (the order of performance was randomly assigned to each participant before each session). The random practice group (high contextual interference) performed 20 trials of each skill randomly assigned, avoiding more than 2 consecutive trials of the same skill. The control group participated in a 30-minute physical activity class. The activi- ties implemented did not include jumping or throwing (e.g. aerobics, walking, running, or weight lifting).

The acquisition test was performed 2 days after the last intervention session. The retention test took place 5 days after the acquisition test. In both tests, the participants were assessed on motor skills performance (jump and throw), in accordance with the test protocol, as in the pretest.

The experimental practice groups (blocked and random) received verbal indications and observational demonstrations of each skill at the beginning of each session; once the participants started their practice, no feedback was given. All experimental sessions were supervised by the principal researcher (physical education teacher). The control group activities were designed and supervised by another physical education teacher, who had been previously trained by the principal investigator.

\section{Statistical analysis}

The data were analysed for a normal distribution. Mean and standard deviation were calculated for descriptive purposes. One-way ANOVA was used to examine between-group differences at pretest. A Pearson correlation analysis was applied to establish the relationship between motor skills performances. Distance jump and overarm throw were analysed separately with a two-way ANOVA with repeated measures in the last factor (3 groups by 3 measurements). The IBM-SPSS ${ }^{\oplus}$ Statistics 23 software was used for all analyses and $p$ values of 0.05 or less were considered statistically significant.

\section{Ethical approval}

The research related to human use has been complied with all the relevant national regulations and institutional policies, has followed the tenets of the Declaration of Helsinki, and has been approved by the authors' institutional review board or an equivalent committee.

\section{Informed consent}

Informed consent has been obtained from all individuals included in this study.

\section{Results}

Normal probability tests (Kolmogorov-Smirnov and Levene) and residual plots indicated that the data were normally distributed $(p>0.05)$. Means and standard deviations for distance jump and overarm throw across all groups are shown in Table 2. 
Table 2. Descriptive statistics for motor skills performance for all groups

\begin{tabular}{|c|c|c|c|c|c|}
\hline \multirow{2}{*}{ Variable } & \multirow{2}{*}{ Group } & \multirow{2}{*}{$\begin{array}{c}\text { Sample } \\
(n)\end{array}$} & Pretest & Acquisition & Retention \\
\hline & & & $M \pm S D$ & $M \pm S D$ & $M \pm S D$ \\
\hline \multirow{3}{*}{ Throw } & CG & 16 & $5.88 \pm 2.45$ & $7.00 \pm 2.31$ & $7.00 \pm 2.13$ \\
\hline & $\mathrm{BP}$ & 18 & $5.94 \pm 3.02$ & $10.50 \pm 1.47$ & $10.44 \pm 1.69$ \\
\hline & $\mathrm{RP}$ & 16 & $5.75 \pm 2.96$ & $9.81 \pm 1.94$ & $9.69 \pm 1.70$ \\
\hline \multirow{3}{*}{ Jump } & CG & 16 & $7.31 \pm 2.77$ & $8.06 \pm 2.32$ & $7.31 \pm 1.78$ \\
\hline & $\mathrm{BP}$ & 18 & $6.44 \pm 1.95$ & $10.44 \pm 1.58$ & $10.33 \pm 1.57$ \\
\hline & $\mathrm{RP}$ & 16 & $6.88 \pm 2.73$ & $9.94 \pm 1.69$ & $9.88 \pm 1.54$ \\
\hline
\end{tabular}

$M$ - mean, $S D$ - standard deviation, CG - control group, BP - blocked practice, RP - random practice

For the pretest data, one-way ANOVA showed no between-group differences at the beginning of the study, either for the jump $\left(F_{(2,47)}=0.51 ; p=0.601\right)$ or for the throw $\left(F_{(2,47)}=0.02 ; p=0.980\right)$. The Pearson correlation analysis indicated no relationship between motor skills performance $(r=0.161 ; p=0.263)$; therefore, both variables were analysed separately. The descriptive data proved low proficiency in the FMS assessed.

For the overarm throw, the two-way ANOVA [group (3) $\times$ measurement (3)], with repeated measures in the last factor, showed a significant interaction $\left(F_{(2,47)}=5.81\right.$; $\left.p=0.001 ; \omega^{2}=4.7 \%\right)$. The main effects for group $(F=$ $8.53 ; p=0.001)$ and measurement $(F=54.21 ; p=0.001)$ were also significant. For the distance jump, the two-way ANOVA also revealed a significant interaction $\left(F_{(2,47)}=\right.$ 10.92; $p=0.001 ; \omega^{2}=7.6 \%$ ). The main effects for group $(F=3.82 ; p=0.029)$ and measurement $(F=10.92$; $p=0.001)$ turned out significant, too.

Post-hoc analyses for the interaction (simple main effects) showed that the performance in the pretest was different from that in the acquisition and retention test in the blocked practice and random practice

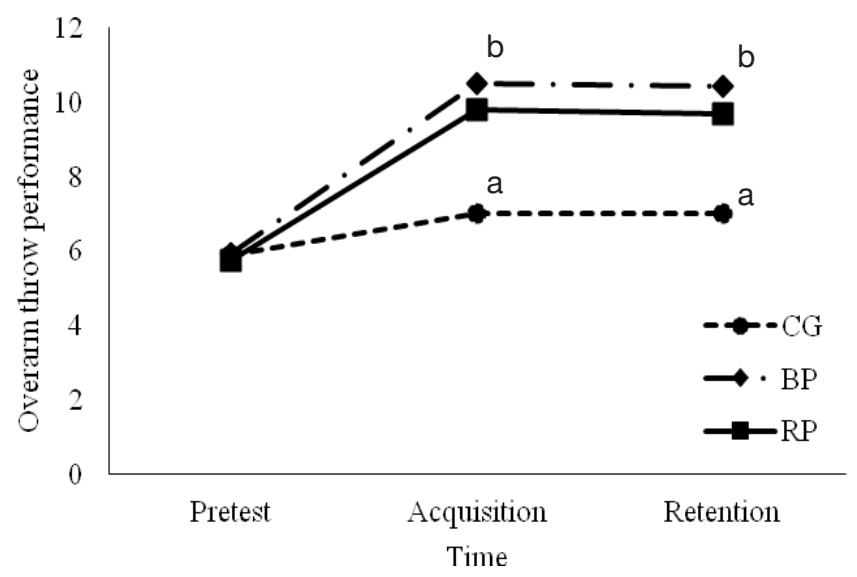

${ }^{a}$ CG different from BP and RP

${ }^{\mathrm{b}}$ Acquisition and retention different from pretest in $\mathrm{BP}$ and $\mathrm{RP}(p<0.05)$.

$\mathrm{CG}$ - control group, BP - blocked practice, RP - random practice

Figure 1. Motor skill performance for overarm throw groups, for throw (Figure 1) and jump (Figure 2). In addition, post-hoc analyses also indicated that the random and blocked practice groups were different from the control group in the acquisition and retention test. No significant difference in performance was found for the control group along the intervention period.

Overall results suggested that both interventions (blocked practice and random practice) helped to improve performance in jump and throw, and maintained performance in the retention test. Physical activity without specific FMS practice did not enhance FMS performance.

\section{Discussion}

The aims of this study were to examine the CIE on FMS performance and to enhance motor performance throughout random and blocked practice on FMS performance in adults. The first hypothesis was based on the CIE, suggesting that blocked practice would have a better performance on acquisition and that random practice would have a better performance

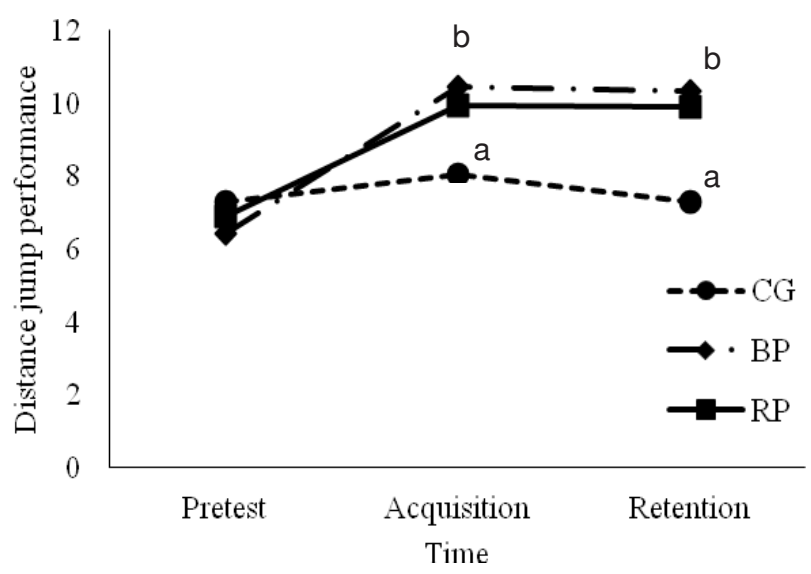

${ }^{a}$ CG different from BP and RP

${ }^{\mathrm{b}}$ Acquisition and retention different from pretest in $\mathrm{BP}$ and $\mathrm{RP}(p<0.05)$. $\mathrm{CG}$ - control group, BP - blocked practice, RP - random practice

Figure 2. Motor skill performance for distance jump 


\section{HUMAN MOVEMENT}

J. Jimenez-Diaz, M. Morera-Castro, W. Salazar, Contextual interference effect on motor skills

on retention. However, the CIE hypothesis was not proved, as no difference between random and blocked practice in acquisition or retention was evidenced. The second hypothesis was supported, showing an improvement of performance in acquisition and retention for both experimental groups.

As for the CIE, the results of the study revealed no difference between the groups, contrary to the typical CIE. In some other studies, there were similar observations regarding analyses of motor performance in applied research settings, for diverse skills and samples [12, 13, 24, 25]. However, the obtained data contradict results that support the CIE $[8,10]$. Li and Wright [8] proved the CIE on motor sequence learning in a laboratory setting, while Wegman [10] found the effect on an FMS in children in applied research.

Regarding the FMS performance, both groups (random and blocked) improved their performance from pretest to acquisition, with similar performance from acquisition to the retention test. These findings are similar to those exposed in previous meta-analytic studies, where random and blocked practice improved performance in acquisition [16]. However, for retention, this study revealed no changes in any experimental group. This behaviour was expected for the random practice group according to Jiménez-Díaz et al. [16], but not for the blocked practice group, in which we awaited a decrease in performance.

The challenge point framework stated by Guadagnoli and Lee [26] suggests that adding difficulty (e.g. assigning random order to the tasks) to a relatively simple task is desired for optimal learning. As the FMS are considered simple tasks for adults, unlike expected by the challenge point, it appears that the extra challenge promoted by random practice did not enhance learning more than assumed in the blocked practice group, illustrating that both practice groups improved and there was no difference in acquisition between them.

In addition, Hebert et al. [15] suggested that in an applied research, blocked practice received more interference than planned; therefore, it behaved similarly to the random practice. Hence, a possible explanation for our findings is that the interference received by the blocked practice group was more than planned and controlled by the investigator, which resulted in no decrease in performance for retention in the group as expected on the basis of a recent meta-analysis outcome [16]. Instead, performance in blocked practice was similar to that in random practice, despite the level of interference during practice. To verify if the setting is a factor that influences the CIE, future stud- ies should explore the CIE on motor skills in applied and laboratory setting, with the same design (adding setting as an independent variable). Also, future research is recommended to add more interference to the practice groups by increasing the number of tasks to be learned or by raising the task difficulty.

The major strength of the present study lies in the effectiveness of random and blocked practice to improve performance of FMS in adults, considering that there is a high prevalence of low FMS performance in this group [20]. In addition, on the basis of the importance of FMS as building blocks of motor performance [27] and its relationship with physical activity [28] and health-related physical fitness [29], we can expect that by improving FMS performance in adults one can enhance physical activity levels and healthrelated physical fitness.

\section{Conclusions}

In conclusion, CIE was not found in FMS performance in adults. However, both types of practice (blocked and random) enhanced FMS learning. Further research should focus on different factors that influence CIE (e.g. task difficulty, research setting, and the number of tasks) to help physical education teachers and coaches when programming practice sessions.

\section{Disclosure statement}

No author has any financial interest or received any financial benefit from this research.

\section{Conflict of interest}

The authors state no conflict of interest.

\section{References}

1. Lee TD, Magill RA. The locus of contextual interference in motor-skill acquisition. J Exp Psychol Learn Mem Cogn. 1983;9(4):730-746; doi: 10.1037/02787393.9.4.730.

2. Shea JB, Morgan RL. Contextual interference effects on the acquisition, retention, and transfer of a motor skill. J Exp Psychol Hum Learn Mem. 1979;5(2):179_ 187; doi: 10.1037/0278-7393.5.2.179.

3. Wright D, Verwey W, Buchanen J, Chen J, Rhee J, Immink M. Consolidating behavioral and neurophysiologic findings to explain the influence of contextual interference during motor sequence learning. Psychon Bull Rev. 2016;23(1):1-21; doi: 10.3758/s13423015-0887-3.

4. Battig WF. Facilitation and interference. In: Bilodeau EA (ed.), Acquisition of skill. New York: Academic Press; 1966; 215-244. 
5. Brady F. A theoretical and empirical review of the contextual interference effect and the learning of motor skills. Quest. 1998;50(3):266-293; doi: 10.1080/0033 6297.1998.10484285.

6. Magill RA, Hall KG. A review of the contextual interference effect in motor skill acquisition. Hum Mov Sci. 1990;9(3-5):241-289; doi: 10.1016/0167-9457(90) 90005-X.

7. Lee TD, Simon DA. Contextual interference. In: Williams AM, Hodges NJ (eds.), Skill acquisition in sport: research, theory and practice. New York: Routledge; 2004; 29-44.

8. Li Y, Wright DL. An assessment of the attention demands during random- and blocked-practice schedules. Q J Exp Psychol A. 2000;53(2):591-606; doi: 10.1080/027249800390628.

9. Wright DL, Magnuson CE, Black CB. Programming and reprogramming sequence timing following high and low contextual interference practice. Res Q Exerc Sport. 2005;76(3):258-266; doi: 10.5641/027013605 x13080719840636.

10. Wegman E. Contextual interference effects on the acquisition and retention of fundamental motor skills. Percept Mot Skills. 1999;88(1):182-187; doi: 10.2466/ pms.1999.88.1.182.

11. Cheong JP, Lay B, Grove JR, Medic N, Razman R. Practicing field hockey skills along the contextual interference continuum: a comparison of five practice schedules. J Sports Sci Med. 2012;11(2):304-311.

12. Cheong JP, Lay B, Razman R. Investigating the contextual interference effect using combination sports skills in open and closed skill environments. J Sports Sci Med. 2016;15(1):167-175.

13. Saemi E, Porter JM, Ghotbi Varzaneh AG, Zarghami M, Shafinia P. Practicing along the contextual interference continuum: a comparison of three practice schedules in an elementary physical education setting. Kinesiology. 2012;44(2):191-198.

14. De Souza MGTX, Nunes MES, Corrêa UC, Santos S dos. The contextual interference effect on sport-specific motor learning in older adults. Hum Mov. 2015;16(3): 112-118; doi: 10.1515/humo-2015-0036.

15. Hebert EP, Landin D, Solmon MA. Practice schedule effects on the performance and learning of low-and high-skilled students: an applied study. Res Q Exerc Sport.1996;67(1):52-58; doi:10.1080/02701367.1996. 10607925.

16. Jiménez-Díaz J, Salazar W, Morera-Castro M. Contextual interference in motor skills performance: a meta-analysis review [in Spanish]. Pensar En Mov Rev Cienc Ejerc Salud. 2016;14(2):1-34; doi: 10.15517/ pensarmov.v14i2.23830.

17. Yanci J, Reina R, Los Arcos A, Cámara J. Effects of different contextual interference training programs on straight sprinting and agility performance of primary school students. J Sports Sci Med. 2013;12(3):601-607.
18. Mazzardo O. Contextual interference: is it supported across studies? Master's thesis. University of Pittsburgh; 2004.

19. Hardy LL, Reinten-Reynolds T, Espinel P, Zask A, Okely AD. Prevalence and correlates of low fundamental movement skill competency in children. Pediatrics. 2012;130(2):e390-e398; doi: 10.1542/peds.2012-0345.

20. Jiménez-Díaz J, Salazar W, Morera-Castro M. Age and gender differences in fundamental motor skills. Pensar En Mov Rev Cienc Ejerc Salud. 2015;13(2):1-16; doi: 10.15517/pensarmov.v13i2.18327.

21. Stodden DF, Langendorfer S, Roberton MA. The association between motor skill competence and physical fitness in young adults. Res Q Exerc Sport. 2009;80(2): 223-229; doi: 10.1080/02701367.2009.10599556.

22. Logan SW, Robinson LE, Wilson AE, Lucas WA. Getting the fundamentals of movement: a meta-analysis of the effectiveness of motor skill interventions in children. Child Care Health Dev. 2012;38(3):305-315; doi: 10.1111/j.1365-2214.2011.01307.x.

23. Jiménez J, Salazar W, Morera M. Design and validation of an instrument to assess fundamental motor skills [in Spanish]. Mot Eur J Hum Mov. 2013;31:87-97.

24. Jones LL, French KE. Effects of contextual interference on acquisition and retention of three volleyball skills. Percept Mot Skills. 2007;105(3 Pt 1):883-890; doi: 10.2466/pms.105.7.883-890.

25. Zetou E, Michalopoulou M, Giazitzi K, Kioumourtzoglou E. Contextual interference effects in learning volleyball skills. Percept Mot Skills. 2007;104(3 Pt 1):9951004; doi: 10.2466/PMS.104.3.995-1004.

26. Guadagnoli MA, Lee TD. Challenge point: a framework for conceptualizing the effects of various practice conditions in motor learning. J Mot Behav. 2004;36(2):212224; doi: 10.3200/JMBR.36.2.212-224.

27. Stodden DF, Goodway JD, Langendorfer SJ, Roberton MA, Rudisill ME, Garcia C, et al. A developmental perspective on the role of motor skill competence in physical activity: an emergent relationship. Quest. 2008;60(2): 290-306; doi: 10.1080/00336297.2008.10483582.

28. Holfelder B, Schott N. Relationship of fundamental movement skills and physical activity in children and adolescents: a systematic review. Psychol Sport Exerc. 2014;15(4):382-391; doi: 10.1016/j.psychsport.2014. 03.005 .

29. Cattuzzo MT, Dos Santos HR, Ré AH, de Oliveira IS, Melo BM, de Sousa Moura M, et al. Motor competence and health related physical fitness in youth: a systematic review. J Sci Med Sport. 2016;19(2):123-129; doi: 10.1016/j.jsams.2014.12.004. 